\title{
Eficiência do turismo nas unidades federativas do Brasil
}

\section{Efficiency analysis of tourism in states of Brazil}

Luan de Oliveira Queiroz - Mestre em Economia pela Universidade Federal de Viçosa. Graduado em Ciências Econômicas pela Universidade Estadual de Montes Claros, UNIMONTES.E-mail: luanqoliveira@gmail.com

Leandro Batista Duarte - Doutorando em Economia pela Universidade Federal de Pernambuco. Mestrado em Economia pela Universidade Federal de Viçosa. E-mail: leandro.duarte1@hotmail.com

Adriano Provezano Gomes - Doutor em Economia Aplicada pela Universidade Federal de Viçosa. Professor Associado do Departamento de Economia da Universidade Federal de Viçosa. E-mail: apgomes@ufv.br

\section{Resumo}

A atividade turística tem demonstrado ser importante para o desenvolvimento socioeconômico mundial, gerando emprego e fonte de riqueza. Deste modo, o presente artigo tem o intuito de identificar as Unidades Federativas Eficientes e Ineficientes quanto à atração de receitas turísticas e fluxo de turistas. A pesquisa foi realizada por meio de dados secundários de órgãos oficiais do turismo e de estudos macroeconômicos, como a Secretaria de Turismo da Bahia, o Ministério do Turismo, o Instituto Brasileiro de Geografia e Estatística e o Banco Central, utilizando como metodologia a abordagem Análise Envoltória de Dados (DEA - Data Envelopment Analysis) que calcula a eficiência relativa e compara as unidades entre si, identificando aquelas de melhor desempenho, uma vez que estas serão parceiras de excelência (benchmarks) para as unidades ineficientes. Nos resultados, observou-se que as unidades federativas que foram tecnicamente eficientes na atração de turistas (Modelo 2) não foram as mesmas quando foram analisadas pela ótica das receitas (Modelo 1), exceto para São Paulo e Espírito Santo, existindo a possibilidade dos estados eficientes no fluxo de turistas aproveitarem este potencial para gerar receitas turísticas a fim de mobilizar dinamicamente sua economia e buscar a eficiência também no cômputo de receitas.

\section{Palavras-chave}

Economia do Turismo. Análise Envoltória de Dados. Eficiência. Brasil.

\begin{abstract}
Tourist activity has shown that is important for the global socio-economic development, creating jobs and wealth source. Thus, this article aims to identify the Efficient and Inefficient Federative Units as the attraction of tourist revenue and flow of tourists. The survey was conducted through secondary data from official tourism bodies and macroeconomic studies, as the Secretary of Tourism of Bahia, the Ministry of Tourism, the Brazilian Institute of Geography and Statistics and the Central Bank, using methodology as the analysis approach Data (ASD - Data Envelopment Analysis) that calculates and compares the relative efficiency of the units to each other, identifying those with improved performance, since they are excellent partners (benchmarks) for the inefficient units. In the results, it was observed that the federal units that were technically efficient in attracting tourists (Model 2) were not the same when analyzed from the standpoint of income (Model 1), except for São Paulo and Espírito Santo, with the possibility of efficient states in the flow of tourists take advantage of this potential to generate tourist revenue to dynamically mobilize its economy and also seek efficiency in computing income.
\end{abstract}

\section{Keywords}

Tourism Economics. Data Envelopment Analysis. Efficiency. Brazil. 


\section{INTRODUÇÃO}

A atividade turística tem demonstrado ser importante para o desenvolvimento socioeconômico mundial, gerando empregos e fonte de riqueza. Segundo o Instituto Brasileiro de Geografia e Estatística (IBGE, 2011), o turismo tem crescido nos últimos anos como um fenômeno econômico e social, razão pela qual as tradicionais descrições dessa atividade, baseadas nos aspectos socioculturais dos visitantes, têm sido complementadas por uma perspectiva de caráter econômico visando identificar e valorar as contribuições geradas por este segmento à economia dos países.

No Brasil, a diversidade das atividades turísticas e a intersecção que estas têm com atividades locais não permitem o isolamento por completo do fenômeno. Dessa forma, separar das atividades características do turismo o volume de gasto realizado pelos visitantes e o gasto realizado pelos residentes constitui-se no maior obstáculo para elaboração de estimativas econômicas consistentes na área (ALVES, 2009, p. 165).

O turismo como atividade econômica possui aspectos que extrapolam a simples reprodução do capital, pois sua expansão implica em mudanças sociais, culturais, políticas e ambientais. Assim, a avaliação dos seus efeitos econômicos deve ser feita de forma multifacetada, o que torna a tarefa ainda mais complexa. Como atividade econômica o turismo possui uma demanda bastante instável, pois o seu fluxo possui uma alta elasticidade em relação à renda e ao preço (ALVES, 2009, p. 165).

A atividade turística, mesmo com toda essa instabilidade, vem ganhando cada vez mais importância no mundo moderno. Desde o final da Segunda Guerra Mundial, como uma das consequências econômicas da estabilidade política trazida pela paz, o turismo evoluiu como atividade, estando sempre associado ao bem-estar e ao aumento da renda das pessoas. Inicialmente, como privilégio de uma minoria, mas, atualmente, como uma atividade de consumo de massa, que se torna cada vez mais acessível para grandes parcelas da população (RUSCHMANN apud ALVES, 2009).

No Brasil, essas atividades turísticas geram uma receita bem significativa, uma vez que o país é bem representado por alguns estados que apresentam um grande fluxo turístico tanto doméstico quanto internacional. Assim, com base em leituras realizadas, levantaram-se dois questionamentos em relação ao tema: (i) Se, tecnicamente, os estados eficientes na atração de turistas também o são em arrecadar receitas advindas das atividades turísticas? (ii) Se, a tendência dos 
escores de eficiência dos estados brasileiros refletem a influência dos agregados econômicos na determinação da variação do fluxo turístico?

$\mathrm{O}$ artigo busca identificar as unidades federativas eficientes e ineficientes quanto à atração de receitas turísticas ou fluxo de turistas, e tem como objetivo específico: Relacionar a tendência dos escores na medida de eficiência dos estados brasileiros com os determinantes ou agregados econômicos do país que afetam a atração de turistas para o país (como o câmbio, a inflação, o PIB, existência de Eventos Internacionais realizados no país, entre outros) na possibilidade de encontrar explicação para estas tendências.

Pretende-se com este estudo analisar a eficiência (utilizando o DEA) dos estados em obter receitas relacionadas aos fluxos turísticos (Nacional e Internacional), oferecendo, dessa forma, informações que contribuam para o melhor conhecimento do mercado turístico e que, de alguma forma, contribuam para orientar a melhoria dos produtos e serviços colocados à disposição dos turistas nos diferentes estados brasileiros.

Este estudo está estruturado da seguinte forma: na introdução, apresentamse o potencial do setor turístico, a justificativa, a pergunta de pesquisa e os objetivos. Na sequência, é mostrada a revisão de literatura que abrange aspectos relacionados aos fatores determinantes do fluxo turístico e da Análise Envoltória de Dados. Na terceira seção, descrevem-se o enquadramento metodológico e os procedimentos da pesquisa. Em seguida, são apresentados e discutidos os resultados da análise dos dados e da avaliação da eficiência dos estados brasileiros quanto ao potencial turístico. Nas considerações finais são apresentadas as conclusões advindas desta pesquisa e tecidas recomendações para trabalhos futuros.

\section{REVISÃO DE LITERATURA}

O turismo, como muitos outros setores, apresenta fatores que o caracterizam como sendo um fenômeno social, pois engloba não somente um viajante isolado, mas sim, diversos aspectos e relações que estão presentes na sociedade como a oferta de bens e serviços para a sua utilização.

Arrilaga (1976, p. 25), ao tratar da definição de turismo, observa que:

O turismo é o conjunto de deslocamentos voluntários e temporais determinados por causas alheias ao lucro; o conjunto de bens, serviços e organização que determinam e tornam possíveis estes deslocamentos, e as relações e fatos que entre aqueles e os viajantes têm lugar. 
O turismo é considerado uma potente força econômica, visto que apresenta fenômenos de consumo; nele origina renda; existem mercados onde se encontram a oferta e a procura; contribui para desenvolver a economia local, regional e nacional; é um importante fator econômico internacional e tem uma verdadeira riqueza política (ARRILAGA, 1976). O turismo possui aspectos microeconômicos e macroeconômicos sendo importante para compreender o comportamento dos seus agentes.

Além disso, o turismo enquanto atividade econômica movimenta fluxos de pessoas gerando altos índices de trocas comerciais, caracterizando regiões de emissão e recepção de turistas, envolvendo na sua dinâmica quatro agentes: o turista, as empresas turísticas, o governo e a comunidade que interagem entre si (PIRES apud SOUZA; DUARTE, 2014).

\subsection{FATORES DETERMINANTES DO FLUXO TURÍSTICO}

Um dos aspectos para esse resultado, de desenvolvimento dessa atividade e dos países, é o fluxo turístico que se caracteriza como um deslocamento de pessoas para determinados lugares, movimentando a economia. Atualmente, os governos nacionais costumam demonstrar grande interesse em monitorar e fixar medidas que avaliem o movimento de entrada e saída de pessoas em seus países.

$\mathrm{Na}$ evolução da história, as viagens sempre foram um componente obrigatório na vida econômica e social dos homens em todas as épocas, distintas para cada civilização (LAGE; MILONE, 2004). Esse deslocamento de pessoas de um local para outro, caracterizado como fluxo, compreende as atividades realizadas no turismo moderno.

Ressalta-se que, para analisar o fluxo turístico, é importante entender alguns dos seus determinantes. Segundo Santos (2004), no destino turístico, o motivo é uma oportunidade de satisfação das necessidades e desejos, pois o turista, que realiza uma viagem a um determinado destino, vê nele algum elemento que the poderá ser positivo, atraente e motivador.

Para a sistematização dos determinantes dos fluxos turísticos é necessária uma divisão. Para Santos (2004) essa divisão é por meio de categoria, a saber: população, sociedade e cultura, aspectos econômicos, motivadores, infraestrutura geral, serviços turísticos, deslocamentos, sistema de distribuição e aspectos legais.

Dentre esses processos, pode-se destacar o fator deslocamento e a evolução técnica na área dos transportes. Conforme Rabahy (2003), o desenvolvimento da aviação comercial trouxe uma grande mudança para os transportes de longa 
distância, constituindo um meio rápido e seguro de transportes de passageiros, além das facilidades de pagamento.

A viagem internacional, a partir dos anos 1950, foi se tornando cada vez mais acessível a uma parcela maior da população devido aos diferentes fatores, como o surgimento do avião a jato para passageiros como resposta ao término da Segunda Guerra Mundial. O desenvolvimento dos meios de transportes e das comunicações foram fatores determinantes que ampliaram as possibilidades de chegar a novas e mais distantes regiões de recebimento ou destino turístico (OMT, 2001).

Desse mesmo modo, o turismo nacional foi favorecido em seu desenvolvimento por fatores similares, como a crescente utilização do automóvel e demais meios de transporte, que melhoraram as possibilidades de mobilidade pessoal e coletiva dentro do próprio país (OMT, 2001).

\subsection{RECEITA TURÍSTICA}

A receita turística pode ser definida como todos os gastos realizados pelos turistas procedentes do país ou do exterior. Segundo Lage e Milone (2004), o Instituto Brasileiro de Turismo (Embratur) determina o saldo entre as receitas e as despesas turísticas no balanço de pagamentos do Brasil com base nos dados estatísticos referentes ao movimento físico do turismo receptivo e emissivo, da permanência média dos turistas e dos gastos médios per capita, não incluindo as despesas com passagens internacionais.

\subsection{O MÉTODO DEA}

A Análise Envoltória de Dados ou Data Envelopment Analysis (DEA) é um instrumento de programação matemática que envolve múltiplos insumos e produtos para avaliar o desempenho das unidades tomadoras de decisão (Decision Making Unit - DMU) entre si (COOPER; SEIFORD; ZHU, 2004). Tal método não paramétrico ${ }^{1}$ permite construir uma medida relativa de eficiência que reflete

\footnotetext{
1 Os modelos de análise de eficiência são considerados não paramétricos porque a partir dos inputs e outputs de cada unidade produtiva, estabelecem uma função de produção que por meio de programação matemática lança um escore de eficiência relativa entre 0 e 1 para estas unidades. Ou seja, assume-se que nem todas as firmas são tecnicamente eficientes. Desta forma, o modelo DEA estima uma fronteira de eficiência, constituída pelas unidades eficientes (coeficiente de eficiência igual a 1), nas quais estas unidades servirão de referência (benchmarks) às unidades consideradas ineficientes (coeficiente de eficiência menor que 1). Diferentemente dos modelos econométricos que formatam seus dados a função de produção teórica estimada e aceitam que todas as firmas sejam eficientes (COELLI et al., 2007; COOPER; SEIFORD; TONE, 2004).
} 
o desvio observado das unidades de produção (DMU) ineficiente em relação a uma fronteira de eficiência onde estão localizadas todas as unidades consideradas eficientes (FERREIRA; GOMES, 2009).

Conceitual e analiticamente a eficiência pode ser classificada conforme os seguintes componentes: (i) Eficiência Técnica: capacidade das firmas em maximizar seu nível de produto dada sua dotação de insumos; (ii) Eficiência de Escala: componente da eficiência técnica que reflete a operacionalização da empresa em escala ótima de produção; (iii) Eficiência Alocativa: consiste na capacidade da firma em utilizar quantidades ótimas de insumo que produzam uma determinada quantidade de produto a um custo mínimo, supondo que sejam conhecidos os preços relativos dos insumos; e Eficiência Econômica ou Custo: que é a combinação da eficiência técnica com a eficiência alocativa, ou seja, a capacidade da firma em obter o máximo produto com o mínimo custo possível, dado o nível de preços vigentes (COELLI et al., 2007; FERREIRA; GOMES, 2009).

A forma de analisar essas medidas de eficiência ainda pode ser separada sobre duas óticas: (i) Orientação Insumo: a avaliação da eficiência fundamentase na redução dos insumos; e (ii) Orientação Produto: a avaliação da eficiência fundamenta-se no aumento do produto (GOMES; BATISTA, 2004).

Empiricamente, os modelos de análise de eficiência mais comumente utilizados são o modelo CCR, desenvolvido por Charnes, Cooper e Rhodes em 1978, e o modelo BCC, de Banker, Charnes e Cooper (1984). O modelo CCR analisa a eficiência produtiva de uma unidade, identificando as fontes de ineficiência e estimando os montantes dessa ineficiência (GOMES; BATISTA, 2004). O modelo BCC constitui uma extensão ao modelo CCR, onde se considera que as unidades avaliadas apresentam rendimentos variáveis à escala, que se podem consubstanciar em rendimentos de escala crescente, caso as variações nos outputs sejam mais do que proporcionais às variações nos inputs, ou em rendimentos de escala decrescente, caso as variações nos outputs sejam menos que proporcionais às variações nos inputs (BANKER et al., 2004). Este modelo devolve uma medida de eficiência técnica, distinguindo entre eficiência técnica pura e a eficiência de escala.

O modelo BCC considera tecnologias com rendimentos variáveis de escala ${ }^{2}$, no entanto, o uso da especificação de retornos constantes resultará em

\footnotetext{
2 Rendimentos de escala podem apresentar os seguintes comportamentos ou tendências: (i) rendimentos constantes de escala (onde as variações nos insumos resultam em variações proporcionais nos produtos); (ii) rendimentos de escala não decrescentes (crescentes e constantes, em que variações nos insumos provocam variações mais que proporcionais nos produtos); e (iii) rendimentos não crescentes de escala constantes e decrescentes, onde variações nos insumos provocam variações menos que proporcionais nos produtos (FERREIRA; GOMES, 2009).
} 
medidas de eficiência técnica que podem ser confundidas com eficiência de escala quando há um conjunto de DMU tendo tamanhos diversos e algumas delas não operando em escala ótima. Entretanto, para atingir a melhor situação para uma DMU, sugere-se combinar os modelos de retornos constantes e variáveis no mesmo conjunto de dados. Caso exista diferença nos valores de eficiência técnica de uma determinada DMU, conclui-se que essa DMU tem ineficiência de escala, que pode ser calculada pela relação entre os valores de eficiência técnica com retornos variáveis e com retornos constantes (BANKER et al., 2004; FERREIRA; GOMES, 2009).

\subsection{ANÁLISE DE JANELA}

A Análise de Janela ou Window Analysis é uma metodologia que possibilita a observação da eficiência das unidades produtivas segundo uma abordagem intertemporal. A utilização deste método permite delinear a tendência dos escores de eficiência das unidades de analise ao longo de tempo, de modo a vislumbrar a performance dos indivíduos temporalmente (COOPER; SEIFORD; ZHU, 2004).

Um dos primeiros estudos a adotar a técnica de avaliar a tendência dos escores de eficiência das DMUs é descrito no trabalho de Charnes et al. (1985). Neste estudo, foram utilizados dados de 14 alas de caça da Força Aérea Americana durante um período de sete meses (COOPER; SEIFORD; ZHU, 2004).

Este procedimento consiste em considerar cada unidade produtiva de um ano como uma DMU diferente, ou seja, o desempenho de uma firma no ano em questão é comparado com sua performance nos outros períodos analisados, considerando, é claro o desempenho das outras unidades de produção nestes períodos (PARADI; YANG; ZHU, 2004). Isto significa que uma mesma DMU em anos diferentes são tratadas como se fossem independentes umas das outras, no entanto não deixa de ser comparável ao longo do tempo (PARADI; YANG; ZHU, 2004). Desta forma, são construídas diferentes fronteiras de referência (janelas) em cada período de tempo e para cada conjunto de DMU. E utilizando o princípio de médias moveis, ou seja, cada vez que se inclui um novo período de tempo na análise, o anterior é retirado, estabelece uma linha de tendência para as DMU (COOPER; SEIFORD; ZHU, 2004).

Neste sentido, a análise janela pode ser considerada um método para o estudo da estabilidade de Modelos de Análise de Eficiência, pois tais análises envolvem a retirada de conjuntos inteiros de observações e sua substituição por outras observações anteriormente não consideradas (COOPER; SEIFORD; ZHU, 2004). 


\section{METODOLOGIA}

Este tópico busca esclarecer a utilização do método adotado para a construção da análise em torno da eficiência técnica e de escala dos estados brasileiros em obter receitas e atrair fluxos turísticos por meio do desenvolvimento de atividades turísticas.

\subsection{DEA - ANÁLISE POR ENVOLTÓRIA DE DADOS}

A DEA permite obter a eficiência de uma DMU (Decision Making Unit ou Unidade Tomadora de Decisão) por comparação com ou em relação às outras DMU incluídas na análise. Uma DMU é considerada eficiente relativamente às outras se sua eficiência atingir o máximo, ou 100\%. As entradas e saídas podem ser mensuradas em unidades completamente diferentes entre si (valor monetário, número de horas, grau de satisfação etc.) (CHARNES et al., 1994; KASSAI, 2002; RAGSDALE, 2004). A Data Envelopment Analysis é uma metodologia baseada em Programação Linear (LP) para executar este tipo de análise. A DEA irá determinar o quão é eficiente uma unidade operacional para converter entradas em saídas quando comparada com outras unidades operacionais (RAGSDALE, 2004, p. 107-109).

\subsection{BANCOS DE DADOS E VARIÁVEIS SELECIONADAS}

Os dados do estudo são provenientes do: (i) Ministério do Turismo, órgão oficial do Brasil responsável por fomentar o desenvolvimento de atividades econômicas ligadas ao turismo; e (ii) de um estudo elaborado pela Fundação Instituto de Pesquisas Econômicas (FIPE), entidade que apoia o Departamento de Economia da Faculdade de Economia, Administração e Contabilidade da Universidade de São Paulo (FEA-USP) e disponibilizado pela Secretaria de Turismo do Estado da Bahia. As variáveis destas instituições utilizadas no trabalho serão descritas a seguir divididas em dois grupos, insumos (input) e produtos (output):

\subsubsection{Insumos}

a) Agências de turismo - Quantidade de empreendimentos que exercem a atividade econômica de intermediação remunerada entre fornecedores e consumidores de serviços turísticos ou os fornece diretamente. 
b) Guias de turismo - Quantidade de profissionais devidamente cadastrados no Instituto Brasileiro de Turismo que exercem as atividades de acompanhamento, orientação e transmissão de informações a pessoas ou grupos, em visitas, excursões urbanas, municipais, estaduais, interestaduais, internacionais ou especializadas.

c) Oferta hoteleira (unidades habitacionais) - Número de unidades habitacionais (quartos, apartamentos e chalés) colocados à disposição dos usuários nos meios de hospedagem.

d) Transportadoras turísticas - Número de empresas que realizam transporte turístico de superfície, caracterizado pelo deslocamento de pessoas em veículos e embarcações por vias terrestres e aquáticas.

e) Destinos Indutores - Quantidade de municípios com maior e melhor infraestrutura e com um conjunto de atrativos qualificados, identificados por meio de pesquisa da consultoria internacional realizada pelo Ministério do Turismo.

\subsubsection{Produtos}

a) Receita turística - Valor arrecadado com o desempenho das atividades turísticas em Reais.

b) Fluxo de turistas - Quantidade de visitantes que viajaram (via aérea ou via terrestre) a um destino fora do seu entorno habitual, por uma duração inferior a um ano, com qualquer motivo principal (lazer, negócios ou outro motivo).

As unidades de análise foram as unidades federativas brasileiras e o corte temporal foi o período de 2008 a 2013. Na modelagem utilizada, cada estado foi considerado uma DMU, ou seja, uma unidade tomadora de decisão. E para calcular a eficiência técnica relativa dessas UF, aplicaram-se modelos DEA de retornos constantes (CCR) e retornos variáveis de escala, BCC, considerando a orientação produto.

É importante salientar que a escolha da orientação produto e do conjunto de imputs deveu-se à acuidade de avaliar a eficiência dos principais itens que influenciam para elevar o potencial turístico de cada estado analisado, com o intuito de contribuir para o aumento do fluxo turístico e da receita para o país. Outro aspecto relevante é que a unidade de análise são os estados brasileiros, o que indica em relação ao objeto de estudo que estes podem estar em diferentes tipos de retorno à escala, sendo interessante, dessa forma, adotar também o modelo de análise de eficiência BCC.

$\mathrm{O}$ artigo dividiu os insumos e os produtos em dois modelos: (i) 1ํㅡodelo: este modelo utilizará dados de três insumos (Agência de Turismo, Oferta 
Hoteleira e Destinos Indutores) e um produto (Receita Turística), uma vez que para a variável receita turística só existem dados registrados para o ano de 2011 e para 19 estados, de acordo com a FIPE; (ii) 2o Modelo: este modelo utilizará dados de três insumos (Agência de Turismo, Oferta Hoteleira e Transportadora Turística) e um produto (Fluxo de Turistas), possuindo dados para os anos de 2008 a 2013 e para todas 27 UF, porém para fim de comparação, a análise foi realizada para as mesmas 19 DMU do Modelo 1 e no ano de 2011, podendo dessa forma compará-los (Quadro 1).

A divisão em dois modelos deve-se em primeiro lugar para poder comparar em 2011 se os estados que foram eficientes quanto à geração de receita turística foi também eficiente na sua capacidade de atrair turistas nacionais e internacionais. E em segundo lugar para obedecer à recomendação de natureza operacional citada nos trabalhos mais recentes que nos modelos DEA, o número de unidades consideradas na análise deve ser no mínimo quatro a cinco vezes superior ao somatório das variáveis insumo e produto, para que o modelo apresente resultados consistentes (FERREIRA; GOMES, 2009).

Quadro 1 - Resumo dos modelos aplicados para análise de eficiência

\begin{tabular}{|c|c|c|c|}
\hline MODELOS & INPUT & OUTPUT & DMU \\
\hline MODELO 1 & $\begin{array}{c}\text { Agência de turismo } \\
\text { Oferta hoteleira } \\
\text { Destinos indutores }\end{array}$ & Receita turística & 19 \\
\hline ANÁLISE & $\begin{array}{c}\text { Agência de turismo } \\
\text { Oferta hoteleira } \\
\text { Destinos indutores }\end{array}$ & Fluxo turístico & 19 \\
DE JANELA & $\begin{array}{c}\text { Agência de turismo } \\
\text { Oferta hoteleira } \\
\text { Transportadoras turísticas } \\
\text { Guias de turismo }\end{array}$ & Fluxo turístico & 27 \\
\hline
\end{tabular}

Fonte: Elaborado pelos autores

Além disso, será aplicada uma Análise de Janela a fim de relacionar a tendência dos escores na medida de eficiência dos estados brasileiros, entre os anos de 2008 e 2013, com os principais agregados econômicos do Brasil que afetam a atração de turistas para o país na possibilidade de encontrar possíveis explicações para estas tendências (Anexo A.3).

\section{RESULTADOS E DISCUSSÃO}

Nesta seção foram discutidos os resultados do estudo. O Quadro 2 ilustra as 19 DMU analisadas quanto a sua eficiência em relação aos modelos aplicados 1 e 2. 
Quadro 2-Resumo das unidades federativas do Brasil consideradas eficientes

\begin{tabular}{|c|c|c|}
\hline Eficiência & Modelo 1 & Modelo 2 \\
\hline $\begin{array}{c}\text { Unidades federativas } \\
\text { tecnicamente eficientes }\end{array}$ & $\begin{array}{c}\text { Bahia, Paraíba, Piauí, } \\
\text { Espírito Santo, São } \\
\text { Paulo e Santa Catarina }\end{array}$ & $\begin{array}{c}\text { São Paulo, Piauí, Espírito } \\
\text { Santo e Distrito Federal }\end{array}$ \\
\hline $\begin{array}{c}\text { Unidades federativas } \\
\text { tecnicamente eficientes que } \\
\text { operam em escala ótima }\end{array}$ & $\begin{array}{c}\text { Bahia, Espírito Santo, } \\
\text { São Paulo, Santa } \\
\text { Catarina }\end{array}$ & $\begin{array}{c}\text { São Paulo, Espírito Santo e } \\
\text { Distrito Federal }\end{array}$ \\
\hline
\end{tabular}

Fonte: Elaborado pelos autores

Ao analisar as DMU usando o modelo DEA_CCR, com orientação produto no Modelo 1 no ano de 2011, concluiu-se que das 19 DMU analisadas, apenas 6 foram tecnicamente eficientes. Percebeu-se, também, que dessas 6, apenas 4 apresentaram medidas de eficiência máxima no modelo DEA_RVE (retornos variáveis de escala), o que indica que operaram sem problema de escala. Esses estados apresentam uma receita bem significativa para o país, uma vez que têm demonstrado grande crescimento no setor turístico, principalmente nos últimos anos. Isso pode ser resultado de investimentos e valorização no turismo por parte de grandes empresários ou do próprio governo.

O turismo em São Paulo não se restringe ao segmento de turismo de negócios. Vários eventos culturais e esportivos atraem milhões de turistas para o estado como são os exemplos do Grande Prêmio no Brasil de Fórmula 1, a Festa do Peão Boiadeiro de Barretos, o Festival de Inverno de Campos do Jordão, entre outros. O turismo de aventuras também se desenvolve em vários municípios, sendo que dois dos destinos mais procurados no Brasil situam-se no estado de São Paulo - Brotas e Socorro. Destaca-se também como forma de turismo ainda no estado paulista, o religioso e o de patrimônio histórico.

Segundo a FIPE (2011), o turismo baiano apresenta-se como uma importante atividade na matriz econômica para a expansão da economia, pois possui uma capacidade de gerar divisas, renda, empregos e impulsionar diferentes segmentos produtivos. Nesse aspecto, a Bahia coloca-se como a segunda Unidade da Federação em ordem de importância do Turismo no seu PIB, representando 5,7\% do PIB total do Estado. A Bahia, porém, mesmo tendo uma estrutura produtiva relativamente diversificada, a participação do Turismo no PIB é maior dentre os estados (FIPE, 2011).

O Espírito Santo destaca pelo seu atendimento ao turista, servindo como recomendação para os demais visitantes. Famosa pelas suas belas praias, hospedagem de qualidade e restaurantes que satisfazem os gostos dos turistas. Já o turismo em Santa Catarina tem se destacado não só como uma atividade de 
importância do estado atualmente, mas também como uma aposta para melhorar o seu desenvolvimento econômico. $\mathrm{O}$ estado apresenta uma receita significativa devido ao grande percentual de turistas estrangeiros, principalmente de visitantes argentinos.

Ao analisar as mesmas 19 DMU que correspondem às UF brasileiras no Modelo 2 no período de 2011, considerando como inputs Agência de Turismo, Oferta Hoteleira e Transportadoras Turísticas e como outputs o Fluxo de Turistas, percebeu-se que apenas 4 estados foram eficientes utilizando o DEA_CCR e com retornos variáveis de escala apenas 3 desses 4 . Vale ressaltar, que algumas DMU apareceram como eficientes considerando o Modelo 2 com fluxo turístico comparado com o Modelo 1, em que o produto foi a receita turística. Destaca-se nesse caso o Distrito Federal.

Dentre os estados considerados eficientes sem problemas de escala no Modelo 2, pode-se destacar apenas São Paulo e Espírito Santo, novamente e mais o Distrito Federal. Quanto ao Distrito Federal, pode-se dizer que não apresenta também problemas de escala, considerada uma DMU eficiente segundo o Modelo 2. Ressalta-se que essa UF apontou um crescimento de mais de 300\% quanto ao número de desembarques aéreos no Aeroporto Internacional de Brasília de 1997 a 2011. Somente em relação aos turistas internacionais, houve um salto de 3.798 passageiros, em 2003, e para 384.392, em 2011. Esses dados podem ser explicados pelo crescimento verificado na elevação da renda dos brasileiros em conjunto com as alterações nas taxas de juros. Além disso, o aumento nas viagens aéreas é explicado pela baixa expressiva na tarifa das passagens. A combinação dessas atividades e das demais relacionadas ao turismo impacta em 1,4\% no PIB do Distrito Federal. Em geral, concluiu-se que o desenvolvimento do setor tem acontecido de maneira gradativa, respeitando as ações de melhoria da infraestrutura turística da cidade e se sensibilizando as alterações econômicas de cada período (SETUR, 2013).

Os estados que foram considerados eficientes constituem-se referência para as demais unidades de análise. Esta referência, conforme a aproximação dos níveis de utilização de insumos e produtos entre os estados brasileiros, pode variar de 0 a 1 . A importância da DMU eficiente para as demais é tanto maior quanto mais próximo de um for a medida de eficiência técnica da DMU objetivo.

O Quadro 3 mostra a tendência dos escores dos estados brasileiros ao longo do tempo através da Análise de Janela no Modelo 2, classificando quanto ao tipo de retorno de escala. 
Quadro 3 - Análise da tendência dos escores de eficiência das UF do Brasil no Modelo 2

\begin{tabular}{|c|c|c|}
\hline $\begin{array}{c}\text { Tendência dos } \\
\text { escores de eficiência } \\
\text { ao longo do tempo }\end{array}$ & Unidades federativas & Tipos de retornos \\
\hline $\begin{array}{c}\text { Tendência em } \\
\text { aumentar sua } \\
\text { eficiência }\end{array}$ & $\begin{array}{c}\text { Amapá, Pará, Rondônia, } \\
\text { Roraima, Alagoas, } \\
\text { Maranhão, Piaú e Rio } \\
\text { Grande do Sul }\end{array}$ & $\begin{array}{c}\text { Apresentam retornos crescentes } \\
\text { (Pará, Rondônia, Roraima, } \\
\text { Maranhão, Piaú e Alagoas), } \\
\text { constantes (Amapá), decrescentes } \\
\text { (Rio Grande do Sul) }\end{array}$ \\
\hline $\begin{array}{c}\text { Tendência em } \\
\text { diminuir sua } \\
\text { eficiência }\end{array}$ & $\begin{array}{c}\text { Paraíba, Bahia, } \\
\text { Pernambuco, Sergipe, } \\
\text { Minas Gerais, Santa } \\
\text { Catarina, Goiás e Mato } \\
\text { Grosso do Sul }\end{array}$ & $\begin{array}{c}\text { Apresentam retornos crescentes } \\
\text { de escala (Paraíba, Sergipe e Mato } \\
\text { Grosso do Sul) e decrescentes } \\
\text { (Bahia, Pernambuco, Minas } \\
\text { Gerais, Santa Catarina e Goiás) }\end{array}$ \\
\hline $\begin{array}{c}\text { Tenter-se constante } \\
\text { em sua eficiência }\end{array}$ & $\begin{array}{c}\text { Tocantins, São Paulo e } \\
\text { Distrito Federal }\end{array}$ & $\begin{array}{c}\text { Apresenta retornos constantes } \\
\text { (Distrito Federal), crescentes } \\
\text { (Tocantins) e decrescentes (São } \\
\text { Paulo) }\end{array}$ \\
\hline $\begin{array}{c}\text { Tendência oscilatória } \\
\text { de sua eficiência }\end{array}$ & $\begin{array}{c}\text { Acre, Amazonas, Ceará, } \\
\text { Rio Grande do Norte, } \\
\text { Espírito Santo, Rio de } \\
\text { Janeiro, Paraná e Mato } \\
\text { Grosso }\end{array}$ & $\begin{array}{c}\text { Apresentam retornos crescentes } \\
\text { (Acre, Amazonas, Ceará, Espírito } \\
\text { Santo, Mato Grosso e Rio } \\
\text { Grande do Norte) e decrescentes } \\
\text { (Rio de Janeiro e Paraná) }\end{array}$ \\
\hline
\end{tabular}

Fonte: Elaborado pelos autores

Observa-se que são 8 estados que possuem uma tendência em aumentar sua eficiência, sendo que Roraima, Pará, Rondônia, Maranhão, Piauí e Alagoas apresentam retornos crescentes, ou seja, se ao longo do tempo aumentarem os inputs, irão aumentar mais que proporcional a quantidade de outputs gerados. Já o Rio Grande do Sul, apesar de ter tendência em aumentar sua eficiência, apresentam retornos decrescentes, ou seja, quando o acréscimo no insumo resulta em aumentos menos que proporcionais na geração de produtos. Por fim, o Amapá apresenta retornos constantes dentre os que possuem tendência em aumentar sua eficiência.

São 8 os estados que possuem tendência em diminuir sua escala, sendo apenas a Bahia, Santa Catarina, Goiás, Pernambuco e Minas Gerais que apresentam tipos de retornos decrescentes. Observa-se que quanto a tendência em manter constante sua eficiência, destacam-se 3 estados, sendo apenas o Distrito Federal com retornos constantes, ou seja, acréscimos no insumo de recursos levam a aumentos proporcionais na quantidade de produtos obtidos. Os estados do Acre, Amazonas, Ceará, Espírito Santo, Rio de Janeiro, Paraná e Rio Grande do Norte 
apresentaram tendências oscilatórias ao longo do tempo analisado, possuindo entres eles os retornos crescentes e decrescentes.

Os agregados econômicos apresentam características observáveis de um fenômeno que altera ou que muda. Algumas variáveis podem influenciar na atração de turistas como a taxa de câmbio e a inflação.

Dentre as diferentes taxas praticadas no mercado de câmbio legal no país, tem-se o "câmbio turismo", visto que é utilizado comumente para classificar as operações relativas à compra e a venda de moeda para viagens internacionais, geralmente em espécie. Nesse contexto, a taxa de câmbio pode influenciar principalmente quanto ao fluxo internacional, reduzindo o número de turistas em determinada região e, consequentemente interferindo na eficiência de alguns estados.

Entre outros indicadores que afetam o turismo, tem-se a inflação como um fator também preocupante no setor turístico. Com o aumento da demanda, por exemplo, por um tipo de bem ou serviço turístico, em especial na época de alta temporada, pode ocorrer uma inflação nos preços dos produtos. O efeito inflacionário sobre o turismo resulta em impactos negativos, pelo fato da demanda turística ser muitas vezes concentrada em pequenos períodos e de os turistas terem uma propensão maior para gastar do que os residentes, nesse caso, há uma tendência de elevação dos preços da região visitada. Desse modo, os bens e os serviços, que são utilizados tanto para os turistas como para os residentes, têm elevação de preços para ambas as categorias de consumidores (IGNARRA, 2003).

\section{CONSIDERAÇÕES FINAIS}

O propósito do trabalho foi dimensionar a Economia do Turismo como área de interesse para ser apoiada por instrumentos científicos. Estes instrumentos ajudam a auxiliar a delineação de políticas públicas no setor de atividades e serviços turísticos mais condizentes com a realidade local, construindo, assim, um plano de desenvolvimento econômico multifacetado e sustentável. O meio para poder destacar este setor no estudo, em particular, foi por meio da avaliação da eficiência técnica dos estados brasileiros quanto à participação da receita e fluxo turístico do país.

Como pode ser percebido no escrito, as unidades federativas que foram tecnicamente eficientes na atração de turistas (Modelo 1) não foram as mesmas quando foram analisadas pela ótica das receitas (Modelo 2), exceto para São Paulo. Ou seja, existe a possibilidade dos estados eficientes no fluxo de turistas aproveitarem este potencial para gerar receitas turísticas a fim de mobilizar 
dinamicamente sua economia e buscar a eficiência também no cômputo de receitas. Outro aspecto que pode ser observado é sobre o interesse de utilizar a orientação insumo. A tentativa foi de demonstrar que os estados que se encontraram na situação de ineficientes nos modelos analisados têm um grande potencial turístico que não está sendo aproveitado eficientemente, dado sua dotação de insumos.

Quanto às tendências dos escores de eficiência dos estados brasileiros, pode-se inferir que para cada estado existem agregados em particulares que influenciam de maneira mais intensa suas atividades turísticas. E o interessante seja então construir estudos e modelos mais complexos que possam correlacionar estes aspectos com a eficiência técnica das unidades de análise de modo particular. Assim, pode-se alcançar resultados bem mais significativos quando o foco for adotar políticas mais bem definidas e especificas para dada unidade de análise.

O sentido do trabalho foi de pontuar a Análise Envoltória de Dados como uma importante ferramenta de análise de eficiência a ser utilizada em estudos de turismo. Além de estimular a utilização dos dados disponíveis, mesmos que escassos, para algo que possa realmente ter significado no âmbito dos tomadores de decisão. Desta forma, é fundamental a ação das unidades federativas e de suas estruturas institucionais e até mesmo da própria universidade para que se realizem estudos para mobilizar e apoiar estas estruturas econômicas de modo a sistematizá-las e organizá-las para que suas ações revelem atividades de alto poder de intervenção na realidade social da região.

\section{REFERÊNCIAS}

ALVES, L. A. de A. Considerações Econômicas a Respeito da Atividade Turística no Brasil. In: SUPERINTENDÊNCIA DE ESTUDOS ECONÔMICOS E SOCIAIS DA BAHIA. Turismo e desenvolvimento na Área de Proteção Ambiental Litoral Norte - Bahia. Salvador: SEI, 2009. p. 165-181. (Série Estudos e Pesquisas, 82)

ARRILLAGA, J. I. de. Introdução ao estudo do turismo: turismo e sociedade. Rio de Janeiro: Editora Rio, 1976.

BANKER, R. D.; COOPER, W. W.; SEIFORD, L. M.; ZHU, J. Returns to scale in DEA. In: COOPER, W. W., SEIFORD, L. M., TONE, K. (Ed.). Handbook on data envelopment analysis. Norwell, Massachusetts: Kluwer Academic Publishers, 2004. p. 40-70. 
COELli, T. J.; RAO, D. S. P.; O’DONNELL, C. J.; BATTESE, G. E. An introduction to efficiency and productivity analysis. 2. ed. New York: Springer, 2007. 349 p.

COOPER, W. W.; SEIFORD, L. M.; TONE, K. (Ed.). Handbook on data envelopment analysis. Norwell, Massachusetts: Kluwer Academic Publishers, 2004, 592p.

COOPER, W. W.; SEIFORD, L. M.; ZHU, J. Data envelopment analysis: history, models, and interpretations. In: COOPER, W. W., SEIFORD, L. M., TONE, K. (Ed.). Handbook on data envelopment analysis. Norwell, Massachusetts: Kluwer Academic Publishers, 2004. P. 01 - 399.

FERREIRA, C. M. C.; GOMES, A. P. Introdução à análise envoltória de dados: teoria, modelos e aplicações. Viçosa: Editora UFV, 2009. 389 p.

FIPE - Fundação Instituto de Pesquisa Econômicas. Caracterização e dimensionamento do turismo receptivo na Bahia. 2011. Disponível em: <http://www.fipe.com.br/web/index.asp>. Acesso em: 28 maio 2014.

GOMES, A. P.; BATISTA, A. J. M. S. Análise envoltória de dados: conceitos e modelos básicos. In: SANTOS, M. L., VIEIRA, W. C. (Ed.). Métodos Quantitativos em Economia. Viçosa: UFV, 2004. p. 121-160.

IBGE - Instituto Brasileiro de Geografia e Estatística. Indicadores. 2011. Disponível em: <http://www.ibge.gov.br/home/estatistica/indicadores/ precos/inpc_ipca/defaultinpc.shtm>. Acesso em: 25 maio 2014.

IGNARRA, L. R. Fundamentos do turismo. 2. ed. rev. e ampl. São Paulo: Pioneira Thompson Learning, 2003.

KASSAI, S. Utilização da Análise por Envoltória de Dados (DEA) na análise de demonstrações contábeis. 2002. 350f. Tese. (Doutorado em Contabilidade e Controladoria) - Universidade de São Paulo, São Paulo, 2002.

LAGE, B. H. G.; MILONE, P. C. Economia do turismo. São Paulo: Atlas, 2004.

MINISTÉRIO DO TURISMO. Dados e fatos: estudos, pesquisas e dados sobre o setor de turismo. 2012. Disponível em: < http://www.turismo.gov.br/ dadosefatos/> Acesso em: 27 maio 2014.

OMT - Organização Mundial do Turismo. Introdução ao turismo. São Paulo: Roca, 2001. 
PARADI, J. C.; YANG, Z.;ZHU, H. Assessing bank and bank branch performance: modeling considerations and approaches. In: COOPER, W. W.; SEIFORD, L. M.; TONE, K. (Ed.). Handbook on data envelopment analysis. Norwell, Massachusetts: Kluwer Academic Publishers, 2004. p. 315-361.

RABAHY, W. A. Turismo e desenvolvimento: estudos econômicos e estatísticos no planejamento. Barueri-SP: Manole, 2003.

RAGSDALE, C. T. Spreadsheet modeling \& decision analysis: a practical introduction to management science. 4. ed. Mason, Ohio: Thomson Learning South-Western, 2004. 842 p.

SANTOS, G. E. de O. Modelo gravitacional do turismo: proposta teórica e estudo empírico dos fluxos turísticos no Brasil. 2004. 176 f. Dissertação (Mestrado) - Escola de Comunicações e Artes, Universidade de São Paulo. 2004

SETUR - Secretaria de Turismo do Distrito Federal. Observatório do Turismo. Brasília, 2013. Disponível em: < http:/ /www.setur.df.gov.br/noticias/ item $/ 2746$-observat $\%$ C3\% B3rio-do-turismo-apresenta-an $\%$ C3\% A 1 liseecon\%C3\%B4mica-do-setor-no-df.html>. Acesso em: 13 jun. 2014.

SOUZA, A. C.; DUARTE, L. B. O Turismo internacional no estado da Bahia. In: SOUZA, Aline Conceição (Org.). Mosaico econômico: do global ao regional. 1. ed. Ilhéus-BA: EDITUS, 2014. p. 39-62. 


\section{Anexos}

Tabela A.1 - Resultados dos modelos com retornos constantes à escala (RCE) e retornos variáveis à escala (RVE), com base no Modelo 1, no ano de 2011

\begin{tabular}{|c|c|c|c|c|}
\hline \multirow[b]{3}{*}{ DMU } & \multicolumn{4}{|c|}{ Orientação Produto } \\
\hline & \multicolumn{4}{|c|}{ Modelo 1} \\
\hline & $\begin{array}{c}\text { Modelo BCC } \\
\text { Eficiência } \\
\text { Técnica }\end{array}$ & $\begin{array}{c}\text { Modelo CCR } \\
\text { Eficiência } \\
\text { Técnica }\end{array}$ & $\begin{array}{l}\text { Eficiência } \\
\text { de Escala }\end{array}$ & $\begin{array}{l}\text { Tipo de } \\
\text { Retorno }\end{array}$ \\
\hline Amazonas & 2,61 & 3,02 & 1,16 & Crescente \\
\hline Pará & 1,71 & 2,33 & 1,36 & Crescente \\
\hline Alagoas & 1,39 & 1,69 & 1,22 & Crescente \\
\hline Bahia & 1,00 & 1,00 & 1,00 & Constante \\
\hline Ceará & 1,13 & 1,19 & 1,05 & Crescente \\
\hline Paraíba & 1,00 & 1,75 & 1,75 & Crescente \\
\hline Pernambuco & 1,03 & 1,08 & 1,05 & Crescente \\
\hline Piauí & 1,00 & 1,31 & 1,31 & Crescente \\
\hline $\begin{array}{l}\text { Rio Grande do } \\
\text { Norte }\end{array}$ & 1,18 & 1,36 & 1,16 & Crescente \\
\hline Espírito Santo & 1,00 & 1,00 & 1,00 & Constante \\
\hline Minas Gerais & 1,86 & 1,93 & 1,04 & Decrescente \\
\hline Rio de Janeiro & 1,95 & 2,29 & 1,18 & Decrescente \\
\hline São Paulo & 1,00 & 1,00 & 1,00 & Constante \\
\hline Paraná & 2,22 & 2,28 & 1,03 & Decrescente \\
\hline Rio Grande do Sul & 2,12 & 2,14 & 1,01 & Decrescente \\
\hline Santa Catarina & 1,00 & 1,00 & 1,00 & Constante \\
\hline Distrito Federal & 1,50 & 1,77 & 1,18 & Crescente \\
\hline Goiás & 1,78 & 1,90 & 1,07 & Crescente \\
\hline $\begin{array}{l}\text { Mato Grosso do } \\
\text { Sul }\end{array}$ & 3,01 & 3,86 & 1,28 & Crescente \\
\hline
\end{tabular}

Fonte: Resultados da pesquisa com base nos dados do Ministério de Turismo 
Tabela A.2 - Resultados dos modelos com retornos constantes à escala (RCE) e retornos variáveis à escala (RVE), com base no Modelo 2, no ano de 2011

\begin{tabular}{|c|c|c|c|c|}
\hline \multirow[b]{3}{*}{ DMU } & \multicolumn{4}{|c|}{ Orientação Produto } \\
\hline & \multicolumn{4}{|c|}{ Modelo 2} \\
\hline & $\begin{array}{c}\text { Modelo BCC } \\
\text { Eficiência } \\
\text { Técnica } \\
\end{array}$ & $\begin{array}{c}\text { Modelo CCR } \\
\text { Eficiência } \\
\text { Técnica } \\
\end{array}$ & $\begin{array}{l}\text { Eficiência } \\
\text { de Escala }\end{array}$ & $\begin{array}{l}\text { Tipo de } \\
\text { Retorno }\end{array}$ \\
\hline Amazonas & 2,55299 & 3,04016 & 1,19 & Crescente \\
\hline Pará & 1,53133 & 2,05000 & 1,34 & Crescente \\
\hline Alagoas & 2,50168 & 3,51547 & 1,41 & Crescente \\
\hline Bahia & 1,89982 & 2,28984 & 1,21 & Decrescente \\
\hline Ceará & 2,21509 & 2,26090 & 1,02 & Crescente \\
\hline Paraíba & 1,00000 & 3,53681 & 3,54 & Crescente \\
\hline Pernambuco & 1,78335 & 1,80256 & 1,01 & Decrescente \\
\hline Piauí & 1,00000 & 1,91287 & 1,91 & Crescente \\
\hline Rio Grande do Norte & 2,54143 & 3,04606 & 1,20 & Crescente \\
\hline Espírito Santo & 1,00000 & 1,00000 & 1,00 & Constante \\
\hline Minas Gerais & 1,19789 & 1,27240 & 1,06 & Decrescente \\
\hline Rio de Janeiro & 1,39498 & 1,61844 & 1,16 & Decrescente \\
\hline São Paulo & 1,00000 & 1,00000 & 1,00 & Constante \\
\hline Paraná & 1,51893 & 1,57687 & 1,04 & Decrescente \\
\hline Rio Grande do Sul & 2,89447 & 2,97555 & 1,03 & Decrescente \\
\hline Santa Catarina & 1,92032 & 1,96289 & 1,02 & Decrescente \\
\hline Distrito Federal & 1,00000 & 1,00000 & 1,00 & Constante \\
\hline Goiás & 1,73027 & 1,76663 & 1,02 & Decrescente \\
\hline Mato Grosso do Sul & 3,45638 & 3,54145 & 1,02 & Crescente \\
\hline
\end{tabular}

Fonte: Resultados da pesquisa com base nos dados do Ministério de Turismo 
Tabela A.3 - Resultados da análise de tendência das UF brasileiras quanto aos escores de eficiência ao longo do tempo, no período de 2008 a 2013

\begin{tabular}{|c|c|c|c|c|c|c|c|c|}
\hline \multirow{2}{*}{ DMU } & \multirow{2}{*}{ Janelas } & \multicolumn{6}{|c|}{ Períodos } & \multirow{2}{*}{ Média } \\
\hline & & P1 & P2 & P3 & P4 & P5 & P6 & \\
\hline \multirow{5}{*}{ Acre } & $\mathrm{J} 1$ & 1,512 & 1,000 & & & & & 1,26 \\
\hline & $\mathrm{J} 2$ & & 1,000 & 2,177 & & & & 1,59 \\
\hline & $\mathrm{J} 3$ & & & 1,089 & 1,269 & & & 1,18 \\
\hline & $\mathrm{J} 4$ & & & & 1,000 & 3,350 & & 2,17 \\
\hline & $\mathrm{J} 5$ & & & & & 3,111 & 3,697 & 3,40 \\
\hline \multirow{5}{*}{ Amapá } & $\mathrm{J} 1$ & 1,762 & 2,768 & & & & & 2,27 \\
\hline & $\mathrm{J} 2$ & & 3,020 & 1,000 & & & & 2,01 \\
\hline & $\mathrm{J} 3$ & & & 1,000 & 1,866 & & & 1,43 \\
\hline & $\mathrm{J} 4$ & & & & 1,000 & 1,692 & & 1,35 \\
\hline & $\mathrm{J} 5$ & & & & & 1,979 & 1,000 & 1,49 \\
\hline \multirow{5}{*}{ Amazonas } & $\mathrm{J} 1$ & 2,949 & 2,532 & & & & & 2,74 \\
\hline & $\mathrm{J} 2$ & & 2,565 & 2,864 & & & & 2,71 \\
\hline & $\mathrm{J} 3$ & & & 2,685 & 2,950 & & & 2,82 \\
\hline & $\mathrm{J} 4$ & & & & 2,907 & 2,478 & & 2,69 \\
\hline & $\mathrm{J} 5$ & & & & & 2,101 & 2,893 & 2,50 \\
\hline \multirow{5}{*}{ Pará } & $\mathrm{J} 1$ & 2,698 & 1,803 & & & & & 2,25 \\
\hline & $\mathrm{J} 2$ & & 1,822 & 2,211 & & & & 2,02 \\
\hline & $\mathrm{J} 3$ & & & 2,095 & 2,109 & & & 2,10 \\
\hline & $\mathrm{J} 4$ & & & & 2,115 & 2,008 & & 2,06 \\
\hline & $\mathrm{J} 5$ & & & & & 1,705 & 2,000 & 1,85 \\
\hline \multirow{5}{*}{ Rondônia } & $\mathrm{J} 1$ & 5,208 & 7,660 & & & & & 6,43 \\
\hline & $\mathrm{J} 2$ & & 7,660 & 4,004 & & & & 5,83 \\
\hline & $\mathrm{J} 3$ & & & 3,293 & 2,741 & & & 3,02 \\
\hline & $\mathrm{J} 4$ & & & & 2,576 & 3,633 & & 3,10 \\
\hline & $\mathrm{J} 5$ & & & & & 3,316 & 3,784 & 3,55 \\
\hline \multirow{5}{*}{ Roraima } & $\mathrm{J} 1$ & 4,344 & 3,173 & & & & & 3,76 \\
\hline & $\mathrm{J} 2$ & & 3,173 & 2,878 & & & & 3,03 \\
\hline & $\mathrm{J} 3$ & & & 1,000 & 1,399 & & & 1,20 \\
\hline & $\mathrm{J} 4$ & & & & 1,719 & 1,000 & & 1,36 \\
\hline & $\mathrm{J} 5$ & & & & & 1,000 & 1,000 & 1,00 \\
\hline \multirow{5}{*}{ Tocantins } & $\mathrm{J} 1$ & 1,080 & 1,000 & & & & & 1,04 \\
\hline & $\mathrm{J} 2$ & & 1,000 & 1,000 & & & & 1,00 \\
\hline & $\mathrm{J} 3$ & & & 1,000 & 1,000 & & & 1,00 \\
\hline & $\mathrm{J} 4$ & & & & 1,000 & 1,322 & & 1,16 \\
\hline & $\mathrm{J} 5$ & & & & & 1,000 & 1,324 & 1,16 \\
\hline
\end{tabular}




\begin{tabular}{|c|c|c|c|c|c|c|c|c|}
\hline \multirow{5}{*}{ Alagoas } & $\mathrm{J} 1$ & 7,246 & 2,873 & & & & & 5,06 \\
\hline & $\mathrm{J} 2$ & & 2,873 & 2,583 & & & & 2,73 \\
\hline & $\mathrm{J} 3$ & & & 2,435 & 3,270 & & & 2,85 \\
\hline & $\mathrm{J} 4$ & & & & 3,283 & 4,063 & & 3,67 \\
\hline & $\mathrm{J} 5$ & & & & & 3,447 & 4,186 & 3,82 \\
\hline \multirow{5}{*}{ Bahia } & $\mathrm{J} 1$ & 1,784 & 1,605 & & & & & 1,69 \\
\hline & $\mathrm{J} 2$ & & 1,844 & 1,706 & & & & 1,77 \\
\hline & $\mathrm{J} 3$ & & & 1,720 & 1,806 & & & 1,76 \\
\hline & $\mathrm{J} 4$ & & & & 1,858 & 1,740 & & 1,80 \\
\hline & $\mathrm{J} 5$ & & & & & 1,805 & 2,277 & 2,04 \\
\hline \multirow{5}{*}{ Ceará } & $\mathrm{J} 1$ & 2,668 & 2,084 & & & & & 2,38 \\
\hline & $\mathrm{J} 2$ & & 2,088 & 2,174 & & & & 2,13 \\
\hline & $\mathrm{J} 3$ & & & 2,211 & 2,250 & & & 2,23 \\
\hline & $\mathrm{J} 4$ & & & & 2,245 & 2,456 & & 2,35 \\
\hline & $\mathrm{J} 5$ & & & & & 2,192 & 2,390 & 2,29 \\
\hline \multirow{5}{*}{ Maranhão } & $\mathrm{J} 1$ & 4,185 & 2,630 & & & & & 3,41 \\
\hline & $\mathrm{J} 2$ & & 2,750 & 3,300 & & & & 3,03 \\
\hline & $\mathrm{J} 3$ & & & 3,300 & 3,047 & & & 3,17 \\
\hline & $\mathrm{J} 4$ & & & & 3,015 & 2,922 & & 2,97 \\
\hline & $\mathrm{J} 5$ & & & & & 2,465 & 2,733 & 2,60 \\
\hline \multirow{5}{*}{ Paraíba } & $\mathrm{J} 1$ & 6,201 & 4,224 & & & & & 5,21 \\
\hline & $\mathrm{J} 2$ & & 4,255 & 4,475 & & & & 4,37 \\
\hline & $\mathrm{J} 3$ & & & 4,474 & 5,777 & & & 5,13 \\
\hline & $\mathrm{J} 4$ & & & & 5,733 & 6,617 & & 6,17 \\
\hline & $\mathrm{J} 5$ & & & & & 5,590 & 8,512 & 7,05 \\
\hline \multirow{5}{*}{ Pernambuco } & $\mathrm{J} 1$ & 1,876 & 1,563 & & & & & 1,72 \\
\hline & $\mathrm{J} 2$ & & 1,566 & 1,689 & & & & 1,63 \\
\hline & $\mathrm{J} 3$ & & & 1,700 & 1,988 & & & 1,84 \\
\hline & $\mathrm{J} 4$ & & & & 2,006 & 2,080 & & 2,04 \\
\hline & $\mathrm{J} 5$ & & & & & 2,019 & 2,217 & 2,12 \\
\hline \multirow{5}{*}{ Piauí } & $\mathrm{J} 1$ & 2,337 & 1,322 & & & & & 1,83 \\
\hline & $\mathrm{J} 2$ & & 1,748 & 1,820 & & & & 1,78 \\
\hline & $\mathrm{J} 3$ & & & 1,785 & 1,700 & & & 1,74 \\
\hline & $\mathrm{J} 4$ & & & & 1,597 & 1,501 & & 1,55 \\
\hline & $\mathrm{J} 5$ & & & & & 1,170 & 1,574 & 1,37 \\
\hline \multirow{5}{*}{$\begin{array}{l}\text { Rio Grande } \\
\text { do Norte }\end{array}$} & $\mathrm{J} 1$ & 3,832 & 2,518 & & & & & 3,18 \\
\hline & $\mathrm{J} 2$ & & 2,518 & 2,665 & & & & 2,59 \\
\hline & $\mathrm{J} 3$ & & & 2,664 & 3,179 & & & 2,92 \\
\hline & $\mathrm{J} 4$ & & & & 3,140 & 3,485 & & 3,31 \\
\hline & J5 & & & & & 2,964 & 3,315 & 3,14 \\
\hline
\end{tabular}




\begin{tabular}{|c|c|c|c|c|c|c|c|c|}
\hline \multirow{5}{*}{ Sergipe } & $\mathrm{J} 1$ & 3,486 & 2,847 & & & & & 3,17 \\
\hline & $\mathrm{J} 2$ & & 3,192 & 2,943 & & & & 3,07 \\
\hline & $\mathrm{J} 3$ & & & 2,918 & 3,584 & & & 3,25 \\
\hline & $\mathrm{J} 4$ & & & & 3,470 & 3,718 & & 3,59 \\
\hline & $\mathrm{J} 5$ & & & & & 3,044 & 4,619 & 3,83 \\
\hline \multirow{5}{*}{$\begin{array}{c}\text { Espírito } \\
\text { Santo }\end{array}$} & $\mathrm{J} 1$ & 1,304 & 1,321 & & & & & 1,31 \\
\hline & $\mathrm{J} 2$ & & 1,331 & 1,146 & & & & 1,24 \\
\hline & $\mathrm{J} 3$ & & & 1,145 & 1,393 & & & 1,27 \\
\hline & $\mathrm{J} 4$ & & & & 1,376 & 1,372 & & 1,37 \\
\hline & $\mathrm{J} 5$ & & & & & 1,165 & 1,241 & 1,20 \\
\hline \multirow{5}{*}{$\begin{array}{l}\text { Minas } \\
\text { Gerais }\end{array}$} & $\mathrm{J} 1$ & 1,205 & 1,073 & & & & & 1,14 \\
\hline & $\mathrm{J} 2$ & & 1,268 & 1,238 & & & & 1,25 \\
\hline & $\mathrm{J} 3$ & & & 1,240 & 1,277 & & & 1,26 \\
\hline & $\mathrm{J} 4$ & & & & 1,209 & 1,246 & & 1,23 \\
\hline & $\mathrm{J} 5$ & & & & & 1,274 & 1,406 & 1,34 \\
\hline \multirow{5}{*}{$\begin{array}{c}\text { Rio } \\
\text { de Janeiro }\end{array}$} & $\mathrm{J} 1$ & 1,404 & 1,241 & & & & & 1,32 \\
\hline & $\mathrm{J} 2$ & & 1,562 & 1,298 & & & & 1,43 \\
\hline & $\mathrm{J} 3$ & & & 1,310 & 1,162 & & & 1,24 \\
\hline & $\mathrm{J} 4$ & & & & 1,235 & 1,288 & & 1,26 \\
\hline & $\mathrm{J} 5$ & & & & & 1,301 & 1,580 & 1,44 \\
\hline \multirow{5}{*}{ São Paulo } & $\mathrm{J} 1$ & 1,000 & 1,000 & & & & & 1,00 \\
\hline & $\mathrm{J} 2$ & & 1,174 & 1,000 & & & & 1,09 \\
\hline & $\mathrm{J} 3$ & & & 1,000 & 1,000 & & & 1,00 \\
\hline & $\mathrm{J} 4$ & & & & 1,000 & 1,000 & & 1,00 \\
\hline & $\mathrm{J} 5$ & & & & & 1,000 & 1,000 & 1,00 \\
\hline \multirow{5}{*}{ Paraná } & $\mathrm{J} 1$ & 1,814 & 1,542 & & & & & 1,68 \\
\hline & $\mathrm{J} 2$ & & 1,811 & 1,811 & & & & 1,81 \\
\hline & $\mathrm{J} 3$ & & & 1,818 & 1,689 & & & 1,75 \\
\hline & $\mathrm{J} 4$ & & & & 1,617 & 1,623 & & 1,62 \\
\hline & $\mathrm{J} 5$ & & & & & 1,677 & 1,618 & 1,65 \\
\hline \multirow{5}{*}{$\begin{array}{l}\text { Rio Grande } \\
\text { do Sul }\end{array}$} & $\mathrm{J} 1$ & 3,849 & 2,964 & & & & & 3,41 \\
\hline & $\mathrm{J} 2$ & & 3,482 & 3,261 & & & & 3,37 \\
\hline & $\mathrm{J} 3$ & & & 3,271 & 3,206 & & & 3,24 \\
\hline & $\mathrm{J} 4$ & & & & 3,061 & 3,010 & & 3,04 \\
\hline & $\mathrm{J} 5$ & & & & & 3,103 & 3,163 & 3,13 \\
\hline \multirow{5}{*}{$\begin{array}{c}\text { Santa } \\
\text { Catarina }\end{array}$} & $\mathrm{J} 1$ & 2,183 & 1,801 & & & & & 1,99 \\
\hline & $\mathrm{J} 2$ & & 2,050 & 2,136 & & & & 2,09 \\
\hline & $\mathrm{J} 3$ & & & 2,140 & 2,176 & & & 2,16 \\
\hline & $\mathrm{J} 4$ & & & & 2,115 & 2,188 & & 2,15 \\
\hline & $\mathrm{J} 5$ & & & & & 2,280 & 2,580 & 2,43 \\
\hline
\end{tabular}




\begin{tabular}{|c|c|c|c|c|c|c|c|c|}
\hline \multirow{5}{*}{$\begin{array}{l}\text { Distrito } \\
\text { Federal }\end{array}$} & $\mathrm{J} 1$ & 1,000 & 1,000 & & & & & 1,00 \\
\hline & $\mathrm{J} 2$ & & 1,000 & 1,000 & & & & 1,00 \\
\hline & $\mathrm{J} 3$ & & & 1,000 & 1,000 & & & 1,00 \\
\hline & $\mathrm{J} 4$ & & & & 1,000 & 1,000 & & 1,00 \\
\hline & $\mathrm{J} 5$ & & & & & 1,000 & 1,000 & 1,00 \\
\hline \multirow{5}{*}{ Goiás } & $\mathrm{J} 1$ & 2,090 & 1,757 & & & & & 1,92 \\
\hline & $\mathrm{J} 2$ & & 1,772 & 1,770 & & & & 1,77 \\
\hline & $\mathrm{J} 3$ & & & 1,772 & 2,033 & & & 1,90 \\
\hline & $\mathrm{J} 4$ & & & & 2,032 & 2,106 & & 2,07 \\
\hline & $\mathrm{J} 5$ & & & & & 2,023 & 2,483 & 2,25 \\
\hline \multirow{5}{*}{$\begin{array}{l}\text { Mato } \\
\text { Grosso }\end{array}$} & $\mathrm{J} 1$ & 3,461 & 2,951 & & & & & 3,21 \\
\hline & $\mathrm{J} 2$ & & 2,973 & 3,216 & & & & 3,09 \\
\hline & $\mathrm{J} 3$ & & & 3,211 & 3,468 & & & 3,34 \\
\hline & $\mathrm{J} 4$ & & & & 3,441 & 3,492 & & 3,47 \\
\hline & $\mathrm{J} 5$ & & & & & 2,983 & 2,823 & 2,90 \\
\hline \multirow{5}{*}{$\begin{array}{c}\text { Mato Grosso } \\
\text { do Sul }\end{array}$} & $\mathrm{J} 1$ & 4,137 & 3,325 & & & & & 3,73 \\
\hline & $\mathrm{J} 2$ & & 3,352 & 3,780 & & & & 3,57 \\
\hline & $\mathrm{J} 3$ & & & 3,777 & 4,210 & & & 3,99 \\
\hline & $\mathrm{J} 4$ & & & & 4,185 & 4,099 & & 4,14 \\
\hline & $\mathrm{J} 5$ & & & & & 3,508 & 5,069 & 4,29 \\
\hline
\end{tabular}

P1=2008; P2=2009; P3=2010; P4=2011; P5=2012; P=2013

Fonte: Resultados da pesquisa com base nos dados do Ministério de Turismo 
